\title{
A General Adjoint Relation for Functional Differential and Volterra Integral Equations with Application to Control ${ }^{2}$
}

\author{
N. H. McClamroch ${ }^{2}$ \\ Communicated by L. Cesari
}

\begin{abstract}
A general adjoint relation is developed between solutions of linear functional differential equations and linear Volterra integral equations. Several useful representations for solutions of such equations arise as a consequence of the adjoint relationship. These representations are then used to obtain directly several results for controlling systems described by either linear functional differential equations or linear Volterra integral equations.
\end{abstract}

\section{Introduction}

In the last several years, several papers have appeared whose main theme has been to investigate various control problems involving systems described by functional differential equations and by Volterra integral equations. Such systems seem to represent an obvious step in the attempt to develop increasingly realistic models in such areas as biology, mechanics, physics, and economics. It is well known that such systems exhibit some rather subtle phenomena; consequently, the resulting mathematics is generally rather involved.

The purpose of this paper is to present a general adjoint relationship between solutions of linear functional differential equations and linear Volterra integral equations; we also indicate several direct applications of this result to some problems in control. This paper is based on the recent paper by Banks (Ref. 1), which was a response to an error in the book by Halanay (Ref. 2). In particular, many of the techniques used in this paper are essentially contained in the paper by Banks and the main result here (Theorem 3.1) can

\footnotetext{
${ }^{3}$ Paper received August 10,1970. This work was supported by the National Science Foundation under Grant No. GK-5798.

2 Assistant Professor, Program in Computer, Information, and Control Engineering, The University of Michigan, Ann Arbor, Michigan.
} 
be viewed as a generalization of one of Banksis main results, Eq. (17). However, the motivation in this work is to present the results in a form suitable for application to control problems.

\section{Assumptions and Notation}

In this section, we present the basic systems to be considered in the paper. The notation is explained and the various assumptions are stated. In addition, we present, without proof, existence and uniqueness results for the systems under consideration.

Use is made of vector and matrix notation throughout the paper. In general, lower case letters denote $n$-vectors and capital letters denote $n \times n$ matrices. A prime denotes matrix transpose. The two basic systems with which we shall be concerned are the linear functional differential equation

$$
\dot{x}_{1}(t)=\int_{a}^{t}\left[d_{s} A(t, s)\right] x_{1}(s)+v_{1}(t)
$$

and the linear Volterra integral equation

$$
x_{2}(t)=-\int_{t}^{b} A^{\prime}(s, t) x_{2}(s) d s-v_{2}(t),
$$

where Eq. (1) is defined on the interval $\left(t_{0}, t_{1}\right]$ and Eq. (2) is defined on the interval $\left[t_{0}, t_{1}\right)$. The two constants $a, b$ satisfy $a \leqslant t_{0}<t_{1} \leqslant b$. We make the following assumptions on the $n \times n$ matrix-valued function $A(t, s)$. The function $A(t, s)$ is Lebesgue measurable on $\left[t_{0}, b\right] \times\left[a, t_{1}\right]$; for each $t \in\left[t_{0}, b\right]$, it is of bounded variation in $s$ and right continuous in $s$ on $\left(a, t_{1}\right)$. We also assume that $A(t, s)=0$ for $t_{0} \leqslant t \leqslant b, a \leqslant s \leqslant t_{1}$ and $t \leqslant s$. We further assume that there is an integrable function $m(t)$ defined on $\left[t_{0}, b\right]$ such that

$$
\begin{aligned}
& \mid A\left(t, s \mid \leqslant m(t) \quad \text { on } \quad\left[t_{0}, b\right] \times\left[a, t_{1}\right],\right.
\end{aligned}
$$

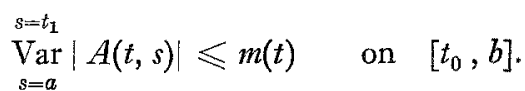

These assumptions are quite weak, but they are sufficient to guarantee that the integrals in (1) and (2) are well defined.

We now consider conditions, under the standing assumptions on $A(t, s)$ just presented, which guarantee that Eqs. (1) and (2) have well-defined and unique solutions. We say that the function $x_{1}:\left[a, t_{1}\right] \rightarrow R^{n}$ is a solution of (1) 
if it is Borel measurable on $\left[a, t_{0}\right]$, absolutely continuous on $\left[t_{0}, t_{1}\right]$, and satisfies (1) almost everywhere on $\left[t_{0}, t_{1}\right]$. A function $x_{2}:\left[t_{0}, b\right] \rightarrow R^{n}$ is a solution of (2) if it is integrable on $\left[t_{1}, b\right]$, of bounded variation on $\left[t_{0}, t_{1}\right]$, and satisfies (2) everywhere on $\left[t_{0}, t_{1}\right)$. Thus, the following theorem is important.

Theorem 2.1. (a) Let $v_{1}:\left[t_{0}, t_{1}\right] \rightarrow R^{n}$ be integrable and let $\tilde{x}_{1}:\left[a, t_{0}\right] \rightarrow R^{n}$ be Borel measurable; there exists a unique solution of (1) which agrees with $\tilde{x}_{1}$ on $\left[a, t_{0}\right]$. (b) Let $v_{2}:\left[t_{0}, t_{1}\right] \rightarrow R^{n}$ be of bounded variation and let $\tilde{x}_{2}:\left[t_{1}, b\right] \rightarrow R^{n}$ be integrable; there exists a unique solution of (2) which agrees with $\tilde{x}_{2}$ on $\left[t_{1}, b\right]$.

The above theorem can be proved using the methods indicated in Refs. 3-4.

The symbols $x_{1}$ and $x_{2}$ have been used to denote the dependent variables in (1) and (2), rather than the more commonly used symbols, in order to preserve a certain symmetry in the equations and also so as not to indicate that either (1) or (2) is primary. Although we have assumed that $a \leqslant t_{0}<t_{1} \leqslant b$, so that (1) is defined for increasing $t$ and (2) for decreasing $t$, the analogous results for $b \leqslant t_{1}<t_{0} \leqslant a$ can easily be obtained.

\section{Main Results}

Under the assumptions stated in the previous section, we proceed to the main results of the paper. Essentially, we show that there is an interesting and useful relationship between solutions of (1) and (2). This result generalizes Eq. (17) in Ref. 1, which was derived for the case where $v_{1}(t)=0$ in (1) and $v_{2}(t)=$ const in (2). This generalization has some interesting consequences, several of which are presented as corollaries.

Theorem 3.1. Let $x_{1}:\left[a, t_{1}\right] \rightarrow R^{n}$ be a solution of (1) and let $x_{2}:\left[t_{0}, b\right] \rightarrow R^{n}$ be a solution of (2), under the assumption that $v_{1}:\left[t_{0}, t_{1}\right] \rightarrow R^{n}$ is integrable and $v_{2}:\left[t_{0}, t_{1}\right] \rightarrow R^{n}$ is of bounded variation. The following equality holds:

$$
\begin{aligned}
& x_{1}^{\prime}\left(t_{1}\right) x_{2}\left(t_{1}\right)+\int_{t_{1}}^{b} x^{\prime}(t) \int_{t_{0}}^{t_{1}}\left[d_{s} A(t, s)\right] x_{1}(s) d t+\int_{t_{0}}^{t_{1}} x_{1}^{\prime}(t) d v_{2}(t) \\
& =x_{1}^{\prime}\left(t_{0}\right) x_{2}\left(t_{0}\right)+\int_{a}^{t_{0}} x_{1}^{\prime}(s) d_{s} \int_{t_{0}}^{t_{1}} A^{\prime}(t, s) x_{2}(t) d t+\int_{t_{0}}^{t_{1}} x_{2}^{\prime}(t) v_{1}(t) d t .
\end{aligned}
$$


Proof. Consider the following string of equalities:

$$
\begin{aligned}
\int_{t_{0}}^{t_{1}} d\left(x_{1}{ }^{\prime}(t) x_{2}(t)\right) & =x_{1}{ }^{\prime}\left(t_{1}\right) x_{2}\left(t_{1}\right)-x_{1}{ }^{\prime}\left(t_{0}\right) x_{2}\left(t_{0}\right) \\
& =\int_{t_{0}}^{t_{1}} x_{1}{ }^{\prime}(t) d x_{2}(t)+\int_{t_{0}}^{t_{1}} x_{1}{ }^{\prime}(t) x_{2}(t) d t .
\end{aligned}
$$

Using (1) and (2), we obtain

$$
\begin{aligned}
& x_{1}^{\prime}\left(t_{1}\right) x_{2}\left(t_{1}\right)-x_{1}{ }^{\prime}\left(t_{0}\right) x_{2}\left(t_{0}\right)=-\int_{t_{0}}^{t_{1}} x_{1}^{\prime}(t) d_{t}\left[\int_{t}^{b} A^{\prime}(s, t) x_{2}(s) d s\right] \\
& \left.\quad-\int_{t_{0}}^{t_{1}} x_{1}{ }^{\prime}(t) d v_{2}(t)+\int_{t_{0}}^{t_{1}} x_{2}{ }^{\prime}(t) \int_{a}^{t} d_{s} A(t, s)\right] x_{1}(s) d t+\int_{t_{0}}^{t_{1}} x_{2}{ }^{\prime}(t) v_{1}(t) d t .
\end{aligned}
$$

But the next to last term can be written as

$$
\begin{aligned}
\int_{t_{0}}^{t_{1}} x_{2}(t) & \int_{a}^{t}\left[d_{s} A(t, s)\right] x_{1}(s) d t \\
= & \int_{t_{0}}^{t_{1}} x_{2}^{\prime}(t) \int_{a}^{t_{1}}\left[d_{s} A(t, s)\right] x_{1}(s) d t=\int_{a}^{t_{1}} x_{1}{ }^{\prime}(s) d_{s} \int_{t_{0}}^{t_{1}} A^{\prime}(t, s) x_{2}(t) d t \\
= & \int_{a}^{t_{0}} x_{1}^{\prime}(s) d_{s} \int_{t_{9}}^{t_{1}} A^{\prime}(t, s) x_{2}(t) d t+\int_{t_{0}}^{t_{1}} x_{1}{ }^{\prime}(s) d_{s} \int_{t_{9}}^{t_{1}} A^{\prime}(t, s) x_{2}(t) d t \\
= & \int_{a}^{t_{0}} x_{1}{ }^{\prime}(s) d_{s} \int_{t_{0}}^{t_{1}} A^{\prime}(t, s) x_{2}(t) d t+\int_{t_{0}}^{t_{1}} x_{1}{ }^{\prime}(s) d_{s} \int_{s}^{b} A^{\prime}(t, s) x_{2}(t) d t \\
& \quad-\int_{t_{1}}^{b} x_{2}{ }^{\prime}(t) \int_{t_{0}}^{t_{1}}\left[d_{s} A(t, s)\right] x_{1}(s) d t,
\end{aligned}
$$

where we have made successive use of the unsymmetric Fubini theorem (Ref. 5) and the property that $A(t, s)=0$ for $s \geqslant t$. Substituting the above into (4) and simplifying yields (3).

Equality (3) is essentially a generalization of the adjoint property for ordinary differential equations, differential difference equations, and integrodifferential equations. Usually, this property is stated for the case where $v_{1}(t)=0$ and/or $v_{2}(t)=$ const. The general form of (3) is useful, as evidenced by the following corollaries. 
Corollary 3.1. Let $v_{1}:\left[t_{0}, t_{1}\right] \rightarrow R^{n}$ be integrable and $\check{x}_{1}:\left[a, t_{0}\right] \rightarrow R^{n}$ be Borel measurable. The corresponding solution of (1) satisfies

$$
x_{1}\left(t_{1}\right)=X_{1}^{\prime}\left(t_{0}\right) \tilde{x}_{1}\left(t_{0}\right)+\int_{a}^{t_{0}}\left[d_{s} \int_{t_{0}}^{t_{1}} X_{1}^{\prime}(t) A(t, s) d t\right] \tilde{x}_{1}(s)+\int_{t_{0}}^{t_{1}} X_{1}^{\prime}(t) v_{1}(t) d t,
$$

where the $n \times n$ matrix-valued function $X_{1}(t)$ is the unique solution of

$$
X_{1}(t)=-\int_{t}^{t_{1}} A^{\prime}(s, t) X_{1}(s) d s+E \quad \text { on } \quad\left[t_{0}, t_{1}\right]
$$

where $E$ is the $n \times n$ identity matrix.

Proof. Let $e^{i}=(0, \ldots, 1, \ldots, 0)$, with 1 in the $i$ th entry for $i=1, \ldots, n$. Consider the $n$ equations

$$
x_{2}^{i}(t)=-\int_{t}^{t_{1}} A^{\prime}(s, t) x_{2}^{i}(s) d s+e^{i}, \quad i=1, \ldots, n .
$$

Clearly, $x_{2}^{i}\left(t_{1}\right)=e^{i}$ for $i=1, \ldots, n$, so that, from Theorem 3.1, with $t_{1}=b$ and $v_{2}^{i}(t)=-e^{i}$, we obtain

$$
\begin{gathered}
e^{i^{\prime}} x_{1}\left(t_{1}\right)= \\
x_{2}^{i^{\prime}}\left(t_{0}\right) \tilde{x}_{1}\left(t_{0}\right)+\int_{a}^{t_{0}} \tilde{x}_{1}^{\prime}(s) d_{s} \int_{t_{0}}^{t_{1}} A^{\prime}(t, s) x_{2}{ }^{i}(t) d t \\
+\int_{t_{0}}^{t_{1}} x_{2}^{i^{\prime}}(t) v_{1}(t) d t, \quad i=1, \ldots, n .
\end{gathered}
$$

Let $x_{2}{ }^{i}(t), i=1, \ldots, n$, form the columns of $X_{1}(t)$; then, in vector-matrix notation, (5) is equivalent to (8) and (6) is equivalent to (7).

Corollary 3.1 gives a representation for the solution of (1) evaluated at $t_{1}$, a result similar to that given in Ref. 1 . A representation for a solution of (2) evaluated at $t_{0}$ can also be given using Theorem 3.1.

Corollary 3.2. Let $v_{2}:\left[t_{0}, t_{1}\right] \rightarrow R^{n}$ be of bounded variation and let $\tilde{x}_{2}:\left[t_{1}, b\right] \rightarrow R^{n}$ be integrable. The corresponding solution of (2) satisfies

$$
x_{2}\left(t_{0}\right)=X_{2}^{\prime}\left(t_{1}\right) \tilde{x}_{2}\left(t_{1}\right)+\int_{t_{1}}^{b} \int_{t_{0}}^{t_{1}} X_{2}^{\prime}(s) d_{s} A^{\prime}(t, s) \tilde{x}_{2}(t) d t+\int_{t_{0}}^{t_{1}} X_{2}^{\prime}(t) d v_{2}(t),
$$

where the $n \times n$ matrix-valued function $X_{2}(t)$ is the unique solution of

$$
\begin{aligned}
& X_{2}(t)=\int_{t_{0}}^{t}\left[d_{s} A(t, s)\right] X_{2}(s) \quad \text { on }\left[t_{0}, t_{1}\right] \\
& X\left(t_{0}\right)=E .
\end{aligned}
$$


Proof. Consider the $n$ equations

$$
\dot{x}_{1}^{i}(t)=\int_{t_{0}}^{t}\left[d_{s} A(t, s)\right] x_{1}^{i}(s), \quad x_{1}^{i}\left(t_{0}\right)=e^{i}, \quad i=1, \ldots, n .
$$

Using Theorem 3.1, with $t_{0}=a$ and $v_{1}^{i}(t)=0$, we obtain

$$
\begin{aligned}
e^{i^{\prime}} x_{2}\left(t_{0}\right)= & x_{1}^{i^{\prime}}\left(t_{1}\right) \tilde{x}_{2}\left(t_{1}\right)+\int_{i_{1}}^{b} \tilde{x}_{2}^{\prime}(t) \int_{t_{0}}^{t}\left[d_{s} A(t, s)\right] x_{1}^{i}(s) d t \\
& +\int_{t_{0}}^{t_{1}} x_{1}^{i^{\prime}}(t) d v_{2}(t), \quad i=1, \ldots, n .
\end{aligned}
$$

Let $x_{1}^{i}(t), i=1, \ldots, n$, form the columns of $X_{2}(t)$; then, in vector-matrix notation, (9) is equivalent to (12) and (10) is equivalent to (11).

Thus, if the $n \times n$ matrix-valued functions $X_{1}(t)$ and $X_{2}(t)$ are known on $\left[t_{0}, t_{1}\right]$, then, for any $v_{1}$ or $v_{2}$ and $\tilde{x}_{1}$ or $\tilde{x}_{2}$, the solutions at $t_{1}$ or $t_{0}$ can be easily determined from the formulas indicated. It is interesting to note that it is not necessary to know $\tilde{x}_{1}, v_{1}$ explicitly to compute $x_{1}\left(t_{1}\right)$ from (5) nor $\tilde{x}_{2}$, $v_{2}$ explicitly to compute $x_{2}\left(t_{0}\right)$ from (9). In fact, the following corollaries of Theorem 3.1, based on results in Ref. 6 , indicate how to compute $x_{1}\left(t_{1}\right)$ or $x_{2}\left(t_{0}\right)$ in terms of simple integrations.

Corollary 3.3. Let $v_{1}:\left[t_{0}, t_{1}\right] \rightarrow R^{n}$ be integrable and let $\tilde{x}_{1}:\left[a, t_{0}\right] \rightarrow R^{n}$ be Borel measurable. The corresponding solution of (1) satisfies

$$
x_{1}\left(t_{1}\right)=z_{1}\left(t_{1}\right)
$$

where $z_{1}:\left[t_{0}, t_{1}\right] \rightarrow R^{n}$ is the absolutely continuous unique solution of the differential system

$$
\begin{aligned}
& \dot{z}_{1}(t)=X_{1}^{\prime}(t) v_{1}(t) \\
& z_{1}\left(t_{0}\right)=X_{1}^{\prime}\left(t_{0}\right) \tilde{x}_{1}\left(\dot{t}_{0}\right)+\int_{a}^{t_{0}}\left[d_{s} \int_{t_{0}}^{t_{1}} X_{1}^{\prime}(\alpha) A(\alpha, s) d \alpha\right] \tilde{x}_{1}(s)_{1} .
\end{aligned}
$$

Proof. Define

$$
z_{1}(t)=X_{1}^{\prime}(t) x_{1}(t)+\int_{a}^{t}\left[d_{s} \int_{t}^{t_{1}} X_{1}^{\prime}(\alpha) A(\alpha, s) d \alpha\right] x_{1}(s),
$$

so that, from Corollary 3.1,

$$
x_{1}\left(t_{1}\right)=z_{1}(t)+\int_{t}^{t_{1}} X_{1}^{\prime}(s) v_{1}(s) d s
$$


for any $t_{0} \leqslant t \leqslant t_{1}$. Hence, $z_{1}$ is absolutely continuous on $\left[t_{0}, t_{1}\right]$. Differentiating the above yields (14). Results (13) and (15) are obtained by direct evaluation.

Corollary 3.4+ Let $v_{2}:\left[t_{0}, t_{1}\right] \rightarrow R^{n}$ be of bounded variation and let $\tilde{x}_{2}:\left[t_{1}, b\right] \rightarrow R^{n}$ be integrable. The corresponding solution of (2) satisfies

$$
x_{2}\left(t_{0}\right)=z_{2}\left(t_{0}\right)
$$

where $z_{2}:\left[t_{0}, t_{1}\right] \rightarrow R^{n}$ is of bounded variation and is the unique solution of the integral system

$$
\begin{aligned}
& z_{2}(t)=z_{2}\left(t_{1}\right)-\int_{t}^{t_{1}} d_{s}\left[\int_{s}^{t_{1}} X_{2}^{\prime}(\alpha) d v_{2}(\alpha)\right] \\
& z_{2}\left(t_{1}\right)=X_{2}^{\prime}\left(t_{1}\right) \tilde{x}_{2}\left(t_{1}\right)+\int_{t_{1}}^{b} \int_{t_{0}}^{t_{1}} X_{2}^{\prime}(s) d_{s} A^{\prime}(\alpha, s) \tilde{x}_{2}(\alpha) d \alpha .
\end{aligned}
$$

\section{Proof. Define}

$$
z_{2}(t)=X_{2}^{\prime}(t) x_{2}(t)+\int_{t}^{b} \int_{t_{0}}^{t} X_{2}^{\prime}(s) d_{3} A^{\prime}(\alpha, s) x_{2}(\alpha) d \alpha,
$$

so that, from Corollary 3.2 ,

$$
x_{2}\left(t_{0}\right)=z_{2}(t)+\int_{t_{0}}^{t} X_{2}^{\prime}(s) d v_{2}(s)
$$

for any $t_{0} \leqslant t \leqslant t_{1}$. Hence, $z_{2}$ is of bounded variation on $\left[t_{0}, t_{1}\right]$ and

$$
z_{2}\left(t_{1}\right)-z_{2}(t)=\int_{t}^{t_{1}} d z_{2}(s)=-\int_{t}^{t_{1}} d_{s}\left[\int_{t_{0}}^{s} X_{2}^{\prime}(\alpha) d v_{2}(\alpha)\right]=\int_{t}^{t_{1}} d_{s}\left[\int_{s}^{t_{1}} X_{2}^{\prime}(\alpha) d v_{2}(\alpha)\right],
$$

so that (17) is obtained. Results (16) and (18) are obtained by direct evaluation.

In Corollary 3.4 , if $v_{2}$ is actually absolutely continuous on $\left[t_{0}, t_{1}\right]$, then $z_{2}$ is absolutely continuous on $\left[t_{0}, t_{1}\right]$, and Eq. (17) can be written in its differentiated form

$$
\dot{z}_{2}(t)=X_{2}^{\prime}(t) \dot{v}_{2}(t)
$$

with $z_{2}\left(t_{1}\right)$ specified in (18). Note that no additional assumption has been made regarding $A(t, s)$, so that $x_{2}(t)$ need not even be continuous, although $z_{2}(t)$ is clearly absolutely continuous if $v_{2}(t)$ has that property. 
It is also clear from (3) that the adjoint relationship between $z_{1}(t)$ and $z_{2}(t)$ is given by

$$
z_{1}^{\prime}\left(t_{1}\right) z_{2}\left(t_{1}\right)+\int_{t_{0}}^{t_{1}} z_{1}^{\prime}(t) d_{t} \int_{t}^{t_{1}} d_{s} \int_{s}^{t_{1}} d v_{2}(\alpha)=z_{1}^{\prime}\left(t_{0}\right) z_{2}\left(t_{0}\right)+\int_{t_{0}}^{t_{1}} z_{2}^{\prime}(t) v_{1}(t) d t .
$$

\section{Some Applications}

We now present several results in control theory which are immediate consequences of the previous corollaries. Corollaries 3.3 and 3.4 are particularly useful since, in a sense, they reduce (1) and (2) to equivalent ordinary differential and integral equations, for which many results in control theory are available. We consider reasonably simple systems in terms of the dependence on the control, although we make no additional assumption on $A(t, s)$. Our results will be stated in terms of the following two linear control systems:

$$
\dot{x}_{1}(t)=\int_{a}^{t}\left[d_{s} A(t, s)\right] x_{1}(s)+B(t) u_{1}(t)
$$

and

$$
x_{2}(t)=-\int_{t}^{b} A^{\prime}(s, t) x_{2}(s) d s-\int_{t}^{t_{1}} B(s) u_{2}(s) d s,
$$

where $B(t)$ is an $n \times m$ matrix-valued function which is bounded and measurable on $[a, b] ; u_{1}$ and $u_{2}$ are $m$-vector valued functions, called control variables, which are integrable on $\left[t_{0}, t_{1}\right]$.

We say that (21) is $R^{n}$-controllable at $t_{1}$ if, for any Borel-measurable function $\tilde{x}_{1}:\left[a, t_{0}\right] \rightarrow R^{n}$ and any $\xi \in R^{n}$, there exists an integrable $u_{1}$ : $\left[t_{0}, t_{1}\right] \rightarrow R^{m}$ such that the corresponding solution of (21) agrees with $\tilde{x}_{1}$ on $\left[a, t_{0}\right]$ and with $\xi$ at $t_{1}$. Similarly, (22) is said to be $R^{n}$-controllable at $t_{0}$ if, for any integrable function $\tilde{x}_{2}:\left[t_{1}, b\right] \rightarrow R^{n}$ and any $\xi \in R^{n}$, there exists an integrable $u_{2}:\left[t_{0}, t_{1}\right] \rightarrow R^{n}$ such that the corresponding solution of (22) agrees with $\tilde{x}_{2}$ on $\left[t_{1}, b\right]$ and with $\xi$ at $t_{0}$. With the methods indicated in Ref. 7 and the results of Corollaries 3.3 and 3.4, the following theorem is obtained.

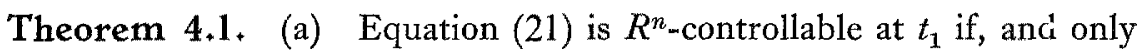
if, the $n \times n$ matrix

$$
W_{1}=\int_{t_{0}}^{t_{1}} X_{1}^{\prime}(t) B(t) B^{\prime}(t) X_{1}(t) d t
$$


is nonsingular. (b) Equation (22) is $R^{n}$-controllable at $t_{0}$ if, and only if, the $n \times n$ matrix

$$
W_{2}=\int_{t_{1}}^{t_{0}} X_{2}^{\prime}(t) B(t) B^{\prime}(t) X_{2}(t) d t
$$

is nonsingular.

We now consider a simple optimal control problem where again the results follow almost directly from Corollaries 3.3 and 3.4. The optimal control problem is of a very simple type for purposes of illustration only; additional complications could easily be introduced.

We say that the control $u_{1}^{*}:\left[t_{0}, t_{1}\right] \rightarrow R^{n}$ is optimal for (21) if the corresponding solution of (21) agrees with $\tilde{x}_{1}$ on $\left[a, t_{0}\right]$ and with $\xi$ at $t_{1}\left(\tilde{x}_{1}\right.$ and $\xi$ are fixed) and if, in addition,

$$
\int_{t_{0}}^{t_{1}} u_{1}^{\prime}(t) u_{1}(t) d t
$$

is a minimum. Similarly, the control $u_{2} *:\left[t_{0}, t_{1}\right] \rightarrow R^{n}$ is optimal for (22) if the corresponding solution of (22( agrees with $\tilde{x}_{2}$ on $\left[t_{1}, b\right]$ and with $\xi$ at $t_{0}\left(\tilde{x}_{2}\right.$ and $\xi$ are fixed $)$ and if, in addition,

$$
\int_{t_{0}}^{t_{1}} u_{2}^{\prime}(t) u_{2}(t) d t
$$

is a minimum. By making use of the methods in Ref. 7 and Corollaries 3.3 and 3.4 , we obtain the following theorem.

Theorem 4.2. (a) If $W_{1}$ is nonsingular, then an optimal control for (21) exists and is given by

$$
u^{*}{ }_{1}(t)=B^{\prime}(t) X_{1}(t) W_{1}^{-1}\left[\xi-X_{1}^{\prime}\left(t_{0}\right) \tilde{x}_{1}\left(t_{0}\right)+\int_{a}^{t_{0}}\left[d_{s} \int_{t}^{t_{1}} X_{1}^{\prime}(\alpha) A(\alpha, s) d \alpha\right] \tilde{x}_{1}(s)\right] .
$$

(b) If $W_{2}$ is nonsingular, then an optimal control for (22) exists and is given by

$$
u_{2}{ }^{*}(t)=B^{\prime}(t) X_{2}(t) W_{2}^{-1}\left[\xi-X_{2}^{\prime}\left(t_{1}\right) \tilde{x}_{2}\left(t_{1}\right)+\int_{t_{1}}^{b} \int_{t_{0}}^{t_{1}} X_{2}^{\prime}(s) d_{s} A^{\prime}(\alpha, s) \tilde{x}_{2}(\alpha) d \alpha\right] .
$$

Theorem 4.1 is essentially an extension of results in Refs. 7-8 to systems described by (21) and (22). Theorem 4.2 extends the optimal control results in Ref. 7 to (21) and (22). Various classes of optimal control problems for systems described by (21) have been studied in Refs. 4, 9, 10; many of those results can be rederived almost trivially using Corollary 3.3. To the author's 
knowledge, control problems associated with systems described by (22) have not been studied previously, except in the special case where $A(t, s)$ is absolutely continuous in $t$ so that (22) can be differentiated to obtain an integrodifferential equation. Results for such problems are given in Refs. 11-12.

Theorems 4.1 and 4.2 follow from Corollaries 3.3 and 3.4. However, various forms of the adjoint relationship given in (3) in Theorem 3.1 are also useful. In particular, the adjoint relationship given in (3) is required to study optimal control problems associated with systems described by (21), if additional constraints on the values of $x_{1}(t)$ on $\left[a, t_{1}\right]$ are specified. Such problems have been studied in Ref. 13, where (3) is used implicitly in the development.

Finally, we mention the possibility of using the results in Corollaries 3.3 and 3.4 as a basis for developing computational algorithms for solving optimal control problems associated with (21) and (22). If one computes $X_{1}(t)$ and $X_{2}(t)$ on $\left[t_{0}, t_{1}\right]$, systems (21) and (22) can be converted to systems of the form (14) and (17), for which well-developed algorithms are available (Ref. 14). Whether such an approach is efficient or not remains to be seen.

\section{References}

1. Banks, H. T., Representations for Solutions of Linear Functional Differential Equations, Journal of Differential Equations, Vol. 5, 1969.

2. Halanay, A., Differential Equation: Stability, Oscillations, and Time Lag, Academic Press, New York, 1966.

3. BANKs, H. T., Necessary Conditions for Problems with Variable Time Lags, SIAM Journal on Control, Vol. 6, No. 1, 1968.

4. Banks, H. T., Variational Problems Involving Functional Differential Equations, SIAM Journal on Control, Vol. 7, No. 1, 1969.

5. Cameron, R. H., and Martin, W. T., An Unsymmetric Fubini Theorem, Bulletin of the American Mathematical Society, Vol. 47, 1941.

6. Bate, A. A., The Optimal Control of Systems with Transport Lag, Advances in Control Systems, Vol. 7, Edited by C. T. Leondes, Academic Press, New York, 1969.

7. Kalman, R. E., Ho, Y. C., and Narendra, K. S., Controllability of Linear Dynamical Systems, Contributions to Differential Equations, Vol. 1, 1963.

8. Weiss, L., On the Controllability of Delay-Differential Systems, SIAM Journal on Control, Vol. 5, No. 4, 1967.

9. Banis, H. T., and JACOBS, M. Q., The Optimization of Trajectories of Linear Functional Differential Equations, SIAM Journal on Control, Vol. 18, No. 4, 1970. 
10. Chyung, D. H., and Lee, E. B., Delayed-Action Control Problems, Automatica, Vol. 6, 1970.

11. Friedman, A., Optimal Control for Hereditary Processes, Archives for Rational Mechanics and Analysis, Vol. 15, 1964.

12. Huang, S. C., Optimal Control Problems with Systems of Integral Equations and Restricted Phase Coordinates (to appear).

13. McClamroch, N. H., Sufficient Conditions for Optimal Control of a General Class of Linear Functional Differential Equations (to appear).

14. Barr, R. O., and GILBERT, E. G., Some Efficient Algorithms for a Class of Abstract Optimization Problems Arising in Optimal Control, IEEE Transactions on Automatic Control, Vol. AC-14, No. 6, 1969. 